\title{
Téoros
}

Revue de recherche en tourisme

\section{Nessipi Kantuet}

\section{Un exemple de tourisme culturel en milieu autochtone}

\section{Michel Noël}

Volume 17, numéro 2, été 1998

L'industrie touristique autochtone

URI : https://id.erudit.org/iderudit/1072274ar

DOI : https://doi.org/10.7202/1072274ar

Aller au sommaire du numéro

Éditeur(s)

Université du Québec à Montréal

ISSN

0712-8657 (imprimé)

1923-2705 (numérique)

Découvrir la revue

\section{Citer cet article}

Noël, M. (1998). Nessipi Kantuet : un exemple de tourisme culturel en milieu autochtone. Téoros, 17(2), 33-35. https://doi.org/10.7202/1072274ar d'utilisation que vous pouvez consulter en ligne.

https://apropos.erudit.org/fr/usagers/politique-dutilisation/ 


\section{UN EXEMPLE DE TOURISME CULTUREL EN MILIEU AUTOCHTONE}

\section{Michel Noël}

Nessipi Kantuet est une entreprise touristique de type fanilial mise sur pied il y a quelques années par Grégoire et Marie-Marthe Gabriel, de la région de Schefferville, ainsi que leur fils Daniel. Grégoire avait fait une longue carrière comme employé de la compagnie minière Iron Ore et Marie-Marthe avait cuvré dans le domaine médical. Daniel, pour sa part, avait acquis une bonne expérience comme guide sur la rivière George. Les membres de la famille Gabriel avaient toujours conservé des liens très étroits avec leur territoire ancestral et ils le fréquentaient régulièrement, soit pour la chasse ou le piégeage, soit lors des vacances. Constatant que des personnes de l'extérieur s'installaient sur leur territoire, ils ont pris conscience qu'ils risquaient de perdre ce territoire convoité, entre autres, par les pourvoiries. Par ailleurs, les parents s'inquiéraient pour l'avenir de leurs enfants et avaient à caur de leur léguer le patrimoine qu'ils avaient eux-mêmes hérité de leurs ancêtres. Ainsi, pour recycler leur territoire et lui donner une nouvelle vocation, ils ont décidé de mettre sur pied une petite entreprise touristique à caractère culturel.

Grégoire et Marie-Marthe Gabriel voyaient dans la mise sur pied d'une telle entreprise de nombreux avantages :

- L'occupation pleine et entière de leur territoire ;

- La création d'emplois pour les membres de la famille et, peut-être, d'autres personnes de la communauté ;

- La revalorisation de leur patrimoine ;

- La possibilité de continuer à être actif dans un domaine qu'ils aimaient, dans un milieu qui leur a toujours été familier :

- La création d'une entreprise dans laquelle ils possédaient déjà une bonne expertise (la vie en forêt) ainsi qu'une base appréciable d'équipements (motoneiges, canots, tentes, chalets, une maison au Lac John et une autre à Schefferville, etc.)
La diffusion culturelle, surtout amenée et soutenue par Daniel - qui, de toute la famille, est le plus étroitement associé à ses parents - s'est révélée une composante des plus originales de l'entreprise. Daniel s'est consacré à la sauvegarde et à la promotion de la culture et du patrimoine de sa communauté innu ; il y a consacré beaucoup de temps et d'énergie et continue sans cesse d'améliorer l'entreprise qu'il a mise sur pied avec Grégoire et Marie-Marthe.

\section{DES DÉBUTS DIFFICILES}

Les débuts de Nessipi Kantuet ont été difficiles. La famille a dû investir une grande partie de ses avoirs personnels dans l'achat d'équipements neufs (dont motoneiges et vêtements d'hiver pour les touristes), la construction de nouveaux chalets, l'acquisition de canots et de mo- teurs, etc. Cependant, le plus difficile ce pourquoi les entrepreneurs touristiques autochtones sont le moins bien préparés également - a incontestablement été la mise en marché de leur produit. Les Gabriel savent que ce produit est original et unique et que les Européens, d'abord, mais aussi les Québécois et les Canadiens, en sont friands. Mais comment rejoindre ces touristes potentiels? Quels sont les moyens et l'aide dont ils peuvent disposer? Les gouvernements accusent un retard en ce qui a trait à l'industrie touristique autochtone. Ils ont peu à offrir aux entrepreneurs en ce qui concerne les infrastructures, l'organisation, le financement et la mise en marché du produit

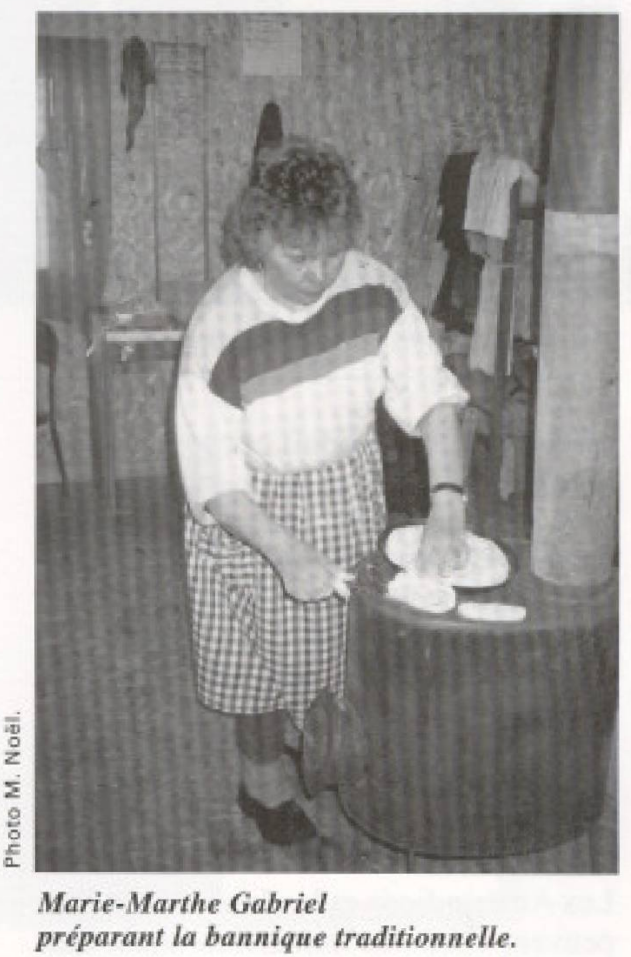




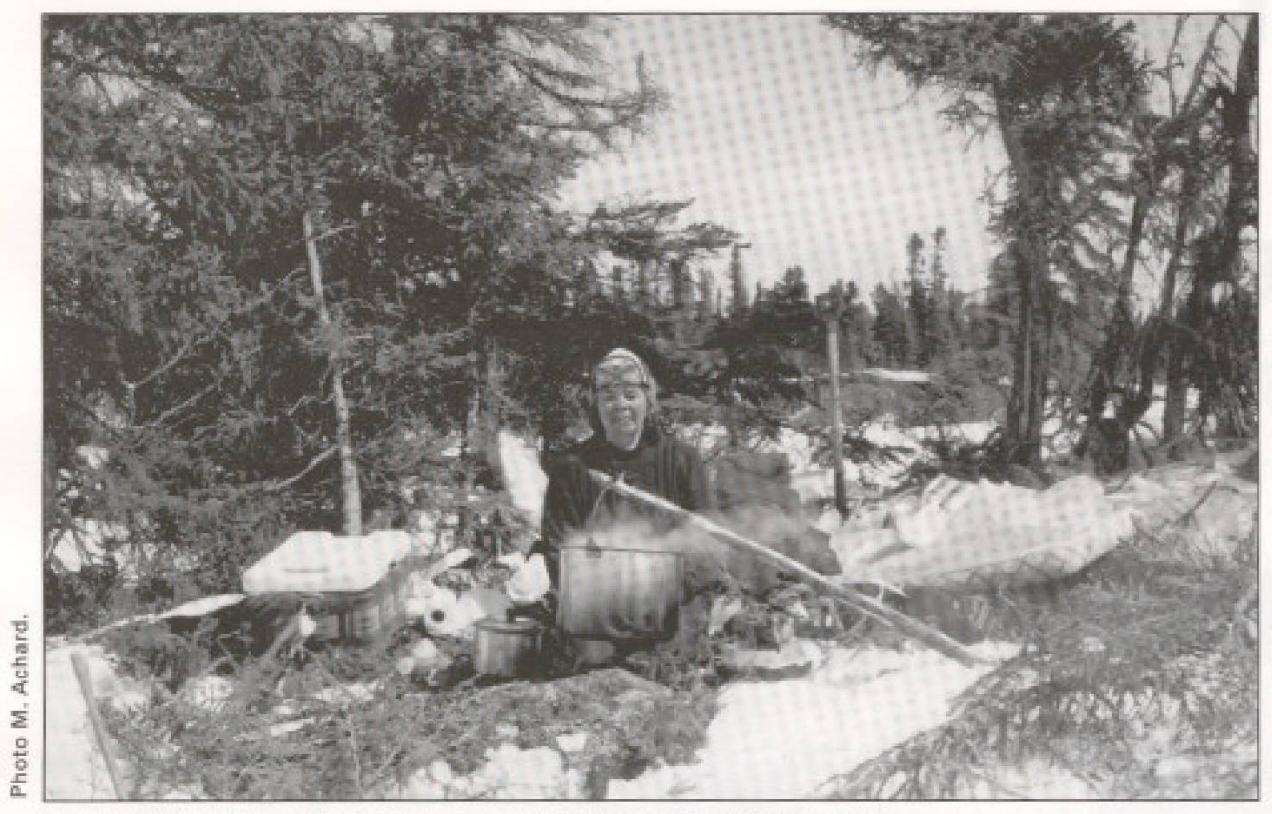

Le repas du midi lors d'une halte d'hiver, région de Schefferville.

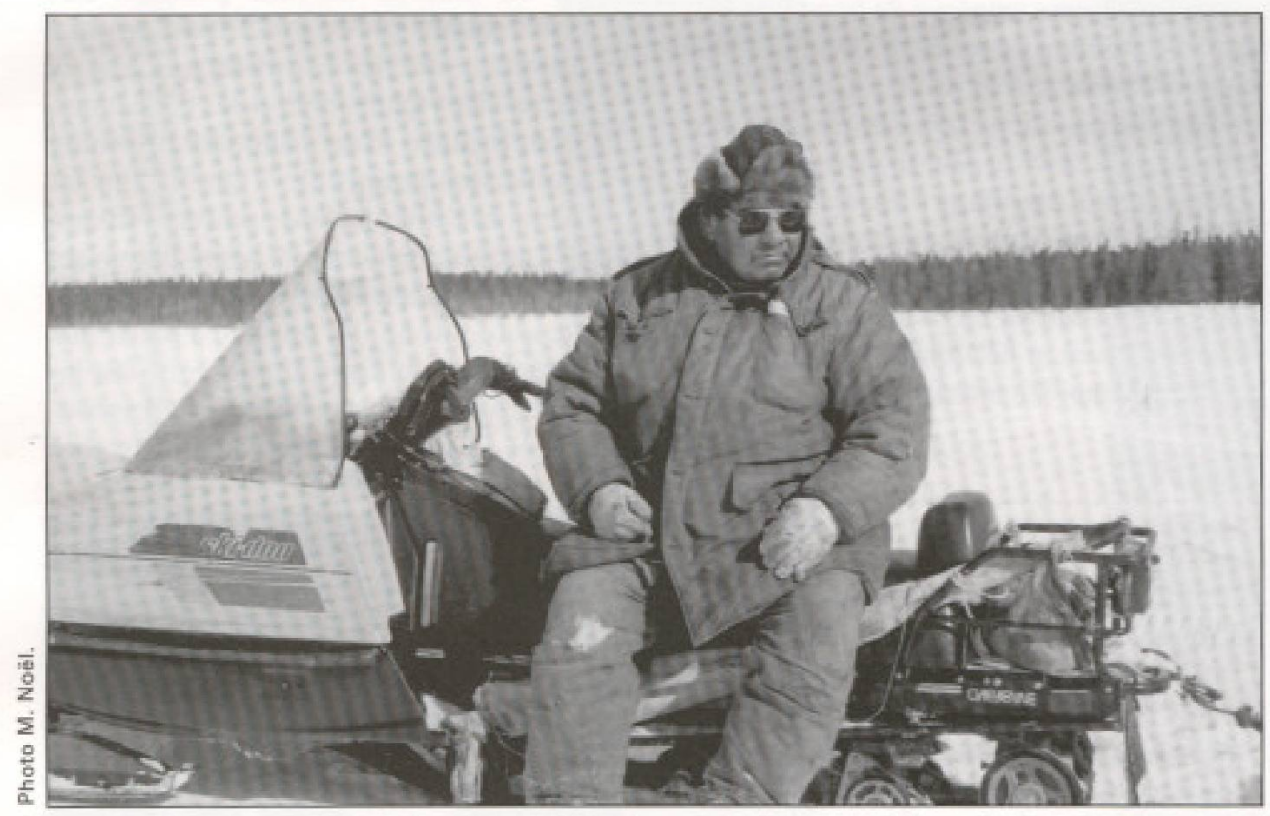

Tourisme d'hiver, Nessipi Kantuet.

touristique. Ce produit est pourtant une ressource exceptionnelle que d'autres provinces canadiennes se sont empressees de valoriser et d'exploiter. Le Québec a l'avantage de pouvoir offrir aux visiteurs un produit touristique autochtone non seulement de qualité, mais aussi d'une très grande authenticité.

\section{L'AUTHENTICITÉ}

Les Amérindiens et les Inuit du Québec peuvent offrir une immersion totale dans bien tracé, parsemé de relais traditionnels (camps-tentes) agréables. L'environnement de la taiga est unique, surtout lorsqu'on le visite en compagnie d'un peuple qui y vit depuis des millénaires. Rapidement, le rythme de vie change. Le dépaysement est total. La confiance, l'amitié et l'entraide règnent. Les activités s'enchaînent sans pour autant qu'il y ait de programme figé ! Paysages, visite de la ligne de trappe, repas communautaire autour d'un feu de bois, nouveau départ, levée des filets à poissons, rencontre d'une famille amérindienne. La nature réserve chaque jour une surprise dont on parlera longtemps : un troupeau de caribous en migration, un ours noir sur la montagne, une volée d'outardes, un pique-nique sur une île au milieu d'un grand lac, la prise d'un poisson exceptionnellement gros... Le soir, les discussions tournent autour de l'histoire des Innu, des légendes, de la spiritualité, de la médecine traditionnelle, de la politique, de l'avenir, des espoirs et des déceptions. Dans ce contexte bien particulier, ce sont les Innu qui parlent euxmêmes de ce qu'ils sont et de ce qu'ils attendent de la vie.

\section{DONNER \\ LA PAROLE AUX INNU}

Le tourisme culturel est réservé à des petits groupes de six à huit personnes, rarement plus; ce n'est pas un tourisme de masse. Il a le grand avantage de donner la parole aux Innu qui se sentent plus à l'aise pour parler et répondre aux questions dans un contexte d'intimité, dans une atmosphère où s'établissent le respect, la compréhension et même la complicité. Ce n'est pas un tourisme passif ou contemplatif ; en effet, le touriste doit participer, être actif, collaborer au bon déroulement du séjour: il conduit sa motoneige, avironne, monte la tente, participe à la corvée du bois de chauffage, peut même, si le couur lui en dit, cuisiner, récolter des plantes médicinales...

C'est le genre de tourisme que Nessipi Kantuet offre aux visiteurs. Je dis * vi= siteurs $w$ car le mot et le concept de * tourisme $* n$ 'existent pas dans la langue innu. Ce sont habituellement des parents ou de très bons amis qui se visitent entre eux et c'est ainsi que le \& touriste $\%$ est accueilli : comme un membre de la famille qui les visite pour une semaine. 


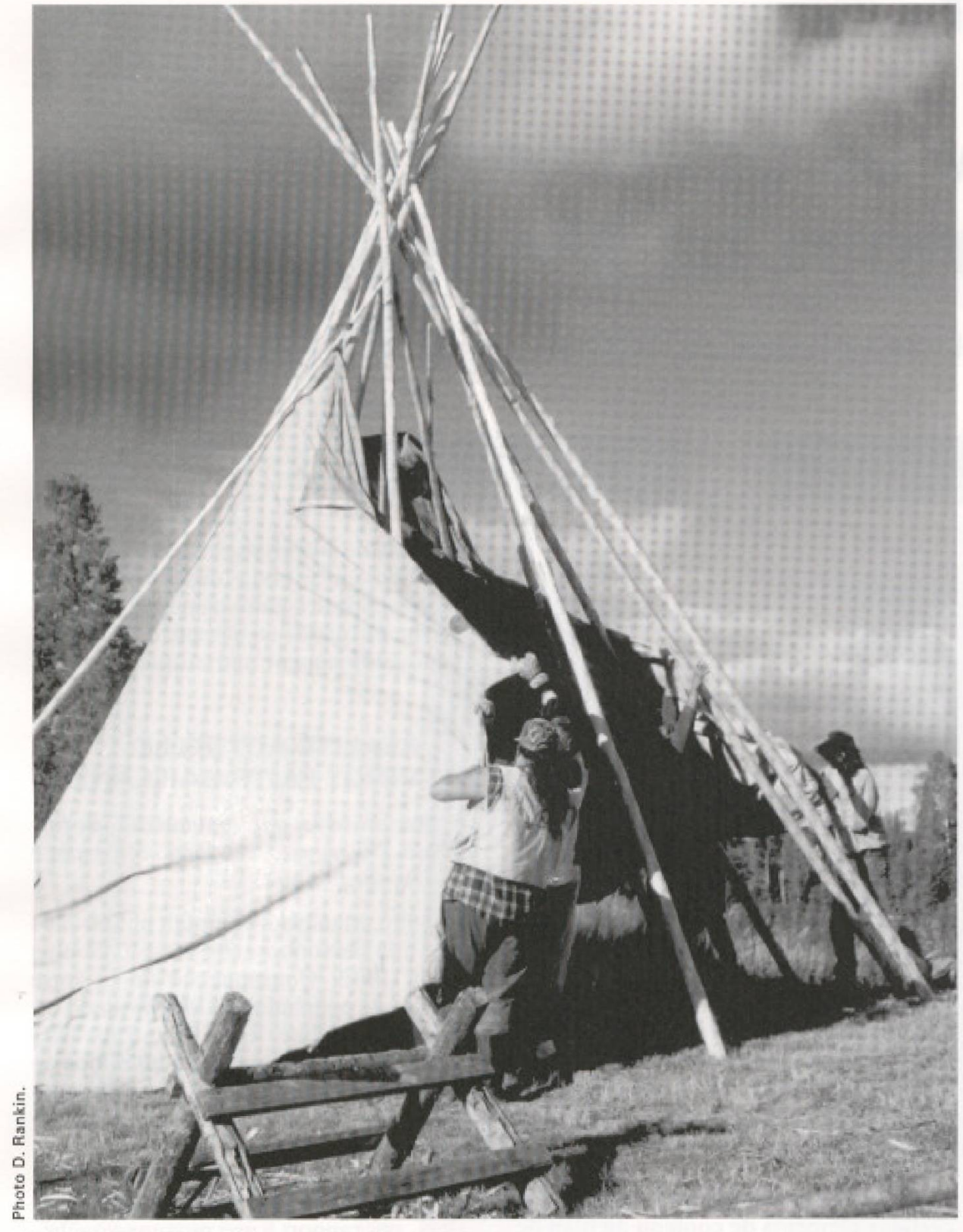

Un tourisme de participation, territoire algonquin.

L'entreprise Nessipi Kantuet existe grâce au courage et à la ténacité de la famille Gabriel. Ils ont cru en ce qu'ils faisaient et ils y ont investi beaucoup de temps, d'argent et d'énergie. Ils ont été au Québec les pionniers du tourisme culturel en milieu autochtone. Leur travail acharné et la qualité de leur produit font qu'ils ont aujourd'hui acquis une réputation enviable. Ils se sont fait connaître en participant à des "salons touristiques", mais c'est surtout le bouche à oreille qui leur a été le plus profitable. D'année en année, la fréquentation augmente. Même si les Gabriel ont franchi de nombreux obsta- ou de « vie inuit $»$ coûte moins cher qu' une semaine de tourisme dans des régions comme Charlevoix, l'Estrie ou la Gaspésie. C'est un mythe de penser que le tourisme autochtone est en soi onéreux ; ce sont les coûts très élevés du transport qui le défavorisent et le rendent inaccessible pour plusieurs.

\section{LA CONCERTATION}

Le tourisme quel qu'il soit ne se développe pas en vase clos. Il nécessite une concertation et une collaboration de tous les instants et à tous les paliers (local, régional, national et international). Il faut « penser * cette industrie qui a d'innombrables ramifications comme une araignée qui tisse sa toile, il faut la développer aussi. Les Amérindiens et les Inuit le savent pertinemment, eux qui souvent habitent des régions éloignées et isolées. Mais cette concertation au Québec et au Canada est au stade embryonnaire II faudrait rapidement y mettre beaucoup d'énergie et, surtout, les ressources nécessaires à sa réalisation. Le défi auquel les promoteurs touristiques font face surtout est l'entraide, car toutes les entreprises, quelles qu'elles soient, bénéficient à court terme des qualités et de la bonne santé des autres. Voilà le défi à relever en cette fin de siècle où les goûts des touristes changent rapidement, où l'industrie touristique doit faire preuve de souplesse et être en mesure de s'adapter à un nouveau * visiteur * qui veut être le " Marco Polo du XXI" siècle »*

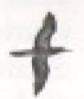

cles, l'avenir de leur entreprise reste précaire. Ils font face à des problèmes de financement, à des tracasseries administratives pour l'obtention de permis et à la concurrence. Le plus grand désavantage se situe au niveau des coûts du transport. C'est d'ailleurs le problème dont est victime tout le Nord du Québec et son développement restera compromis tant et aussi longtemps que les gouvernements et les instances régionales n'y auront pas trouvé une solution. Ainsi, il en coûte aussi cher voyager de Montréal à Madagascar ou à Sydney que de Montréal à Povirnituk (dans le Nord du Québec). Pourtant, une fois sur place, une semaine de vie à l'amérindienne

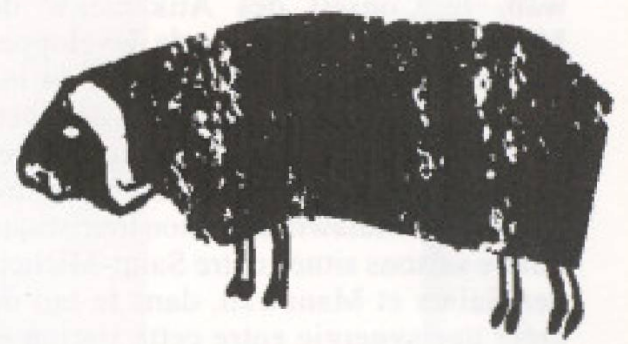

TÉOROS / ÉTÉ 1998 